\title{
UNVEILING ALGAL BIODIVERSITY OF EL- FARAFRA OASIS (WESTERN DESERT, EGYPT) AND POTENTIAL RELEVANCE OF ITS USE IN WATER BIO-ASSESSMENT: SPECIAL INTEREST ON SPRINGS AND DRILLED WELLS
}

\author{
Abd El-Salam M. Shaaban, Hoda A. Mansour, Abdullah A. Saber* \\ Botany Department, Faculty of Science, Ain Shams University, Abbassia-11566, Cairo, \\ Egypt
}

\begin{abstract}
For the first time, comprehensive phycological screening on El-Farafra Oasis (Western Desert, Egypt) had been implemented for unveiling and characterization of its cryptic algal diversification, with special interest on springs and drilled wells. Overall during summer 2011 and winter 2012, 82 different algal taxa have been found in all studied habitats as naturally-grown and culture-induced propagations. Of these, different 14 algal strains have been recorded only using water-culturing techniques on different four algal media (Kolkwitz's solution, Chu \#10, BBM and modified BG-11 media). Culturing techniques not only permitted to discover the cryptic algal taxa present in the viable spores state, but also allowed to follow and confirm the complete life cycle of the rare stigonematalean true T-branched cyanoprokaryote Westiellopsis prolifica. This inventory enriched the freshwater Egyptian algal flora with 6 new algal taxa for the first time namely Symploca dubia, Westiellopsis prolifica, Euastrum insulare, Penium margaritaceum, Zygnemopsis sp. and Nitellopsis obtusa. In this study, some recorded taxa are considered to be crenophilous, e.g. Geitlerinema jasorvense and Phormidium carboniciphilum, to be crenoxenic e.g. Jaaginema subtilissimum, Phormidium corium, Westiellopsis prolifica and Zygnemopsis sp. Only, Oscillatoria tenuis had the highest-grade constancy of occurrence throughout the whole investigation period. This species shows a strong $\alpha$-mesosaprobic indication for water eutrophication in this oasis. In general, El-Farafra Oasis wells and springs are considered isolated and "azonal" habitats still bearing partially near-natural conditions with some agricultural, pastures and humans impacts, and have freshwater type with slight seasonal variations in their mineralization contents as a kind of ecotonescharacteristic features. Finally, these hyper-arid-desert-dwelling water systems are just about excellent potable water resources in the context of water quality guidelines of Egyptian Higher Committee for Water (EHCW) and World Health Organization (WHO), but they substantially still need special governmental efforts in the future to mainly eliminate the relatively high contents of $\mathrm{Fe}, \mathrm{Mn}$ and $\mathrm{Zn}$.
\end{abstract}

Keywords: Algal diversity, culture, springs, drilled wells, bio-monitoring, El-Farafra Oasis, Egypt.

* Correspondent author: email: abdullah_elattar@sci.asu.edu.eg

(ISSN: 1110-8649) 


\section{Introduction}

Over the last century and the start of this millennium, freshwater phycological research in Egypt was traditionally concerned with the main course of the River Nile and its two main branches, with some conspicuous concern on lakes, pools and agricultural drainages, but more or less disregarding cryptic algal biodiversity of the remote geographically-isolated habitats (e.g., Shaaban, 1994; Hamed, 2005, 2008; Saber, 2010). Literature specifically devoted to algal assemblages and species composition of the Egyptian oases and their relatively comparable habitats was very limited (e.g., Shaaban and El Habibi, 1978; Gharib, 2004; Fawzy et al., 2013 and Hifney et al., 2013 in El-Kharga Oasis; Shaaban, 1985 in Siwa Oasis; Shaaban et al., 1997 and Ibraheem and Al-Sherif, 2009 in ElBahariya Oasis; Shaaban and Hamed, 1997 in El-Arish Valley and its vicinity (North Sinai); Hamed, 2005 \& 2008 interested in diatoms and cyanobacteria from some hot springs and other different habitats; Piatek et al., 2009 investigated chrysophyte stomatocysts at Ain Sukhna). Simply, El-Farafra Oasis is one of the aforementioned places that didn't undergo before for any comprehensive phycological study. This is in spite of having attracted the attention of many investigators in other scientific research fields including: geology, hydrogeology, geochemistry, hydrochemistry, biostratigraphy, archaeology, fossil plant remains, micropaleontology, socio-economic aspects, higher plant flora, and entomology (e.g., Ali, 2002; Abd El-Ghani and Fawzy, 2006; Kamal El-Din et al., 2006; Puchnarewicz, 2006; Imam, 2007; ElBastawesy and Ali, 2011; Saafan et al., 2011).

Worldwide, algal flora of ambient and hot springs; in addition to other comparable habitats "drilled wells" (e.g., Uher et al., 2001; Mohamed and AlShehri, 2008), had been the main objectives of many researchers (Cantonati $\boldsymbol{e t}$ al., 2006, 2012 a, b \& c, 2014, 2015; Spitale et al., 2012; Kaštovský et al., 2014), also in the arid regions (Aboal et al., 1998; Amarouche-Yala et al., 2014). Springs (and drilled wells) tend to be relatively isolated and "azonal" water systems (Keleher and Rader, 2008). These unique ecosystems have special interesting characteristics (Cantonati $\boldsymbol{e t}$ al., 2006) and are considered oligotrophic (Kociolek and Stoermer, 2009) headwaters, particularly important for the ecosystem services they provide (Lowe and Likens, 2005) and for conservation of aquatic biodiversity (Cantonati et al., $2012 \mathrm{c}, \mathbf{2 0 1 5}$ ). However, they are mainly threatened by water exploitation (capturing), but have the potential to contribute to the conservation of imperiled freshwater algal biodiversity (Sherwood and Sheath, 1999; Cantonati et al., 2012c; 2015). Unfortunately, springs are mostly neglected in governmental conservation legislation (Cantonati et al., 2007b).

Based on the classification system adopted by Glazier (2009), springs (and other comparable habitats "wells") characterized by a temperature below the mean 
annual air temperature (MAAT) is called "cold springs". Ambient springs are approaching the MAAT in the drainage basins. Thermal springs have water temperatures clearly higher than MAAT. While hot springs have temperatures exceeding the human-body one.

The main aims of the present work were manifold: (1) to unveil and characterize as many as possible algal biocenosis of different watercourses in ElFarafra Oasis, especially those inhabiting springs and drilled wells, (2) bioassessment of water quality based on the resultant data, (3) characterization of the main decisive environmental drivers responsible for algal species composition and distribution, (4) to which extent the oasis watercourses are considered potable for human drinking, (5) finally, suggesting the most appropriate foundations to achieve the maximum utilization of these isolated desert-dwelling freshwater ecosystems.

\section{Materials and Methods}

Study area: El-Farafra Oasis $\left(26^{\circ} 00^{\prime \prime}-27^{\circ} 30^{\prime \prime} \mathrm{N}, 27^{\circ} 20^{\prime \prime}-29^{\circ} 00^{\prime \prime} \mathrm{E}\right)$ is an irregular, triangle shape-cut in a white chalk bed, and bounded by steep cliffs on three sides. It is located in the middle part of the Western Desert (Egypt) nearly at about $650 \mathrm{~km}$ to the southwest of Cairo (Map 1). It approximately covers an area over $10,000 \mathrm{~km}^{2}$ (El-Bastawesy and Ali, 2011; Saafan et al., 2011). This natural depression is located in a hyper-arid region (Ayyad and Ghabbour, 1986) and characterized by hot desert climate with mean annual air temperature $\left(\mathrm{ca} .22^{\circ} \mathrm{C}\right.$ ) and average annual precipitation of about $2 \mathrm{~mm}$. year ${ }^{-1}$ (http://en.wikipedia.org/wiki/Farafra,_Egypt). The solely freshwater main source in the oasis is the largest underground renewable freshwater reservoir (Nubian Sandstone Aquifer System) known in the whole Western Desert (Bornkamm and Kehl, 1990).

Till 2011, there are about 215 drilled wells "also called Birs" widely distributed throughout El-Farafra Oasis with different pumping systems (natural water pressure-pumping or motor-pumping systems). However, there are only 4 springs "also called Ains in arabic" mainly concentrated in Qasr El-Farafra (Ain El-Balad, Ain El-Hateyya, Ain Bishwa and Ain Goshna). The first one is the only slightly hot spring, whilst the others are ambient-to-thermal stagnant springs with motors-driven water systems (Mohammed Abd El-Sattar, Director of Inspection Groundwater in El-Farafra Oasis, personal communication, 2011; Saber, personal observation). 


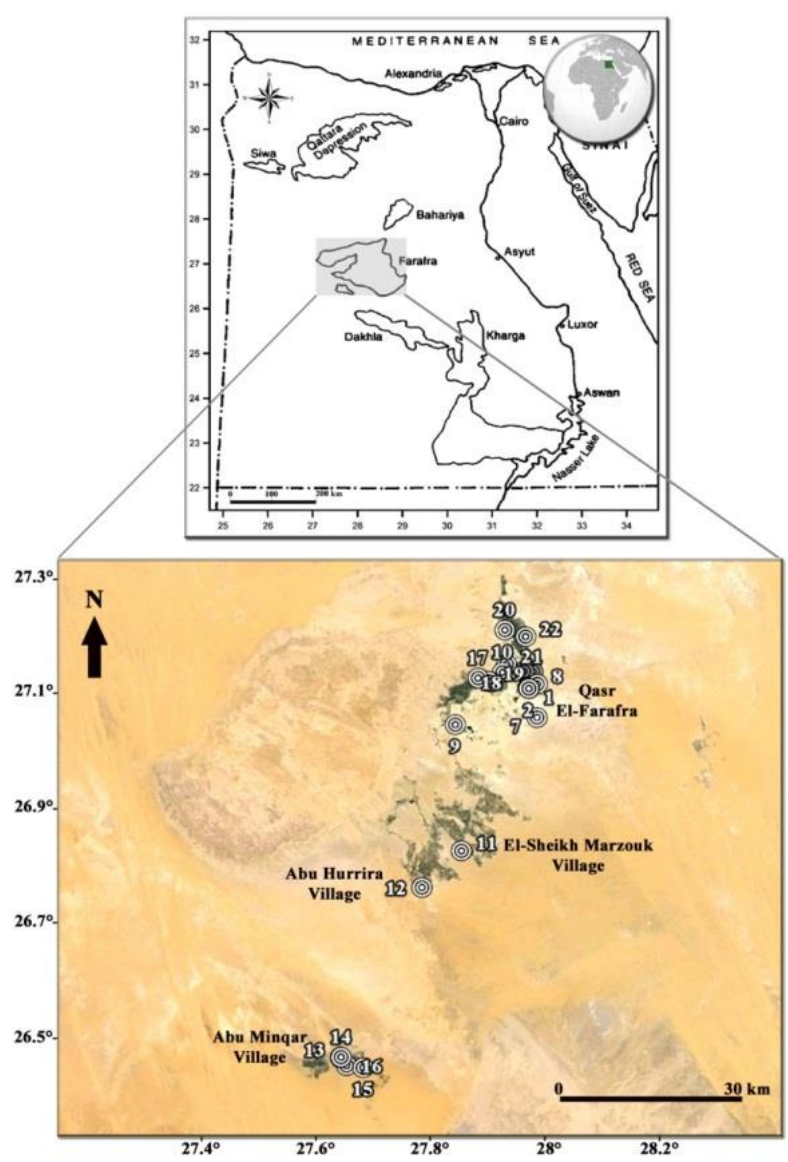

Map 1. Stations of materials collection from El-Farafra Oasis, Western Desert (Egypt). 1= Ain ElBalad (Qasr El-Farafra), 2 = Agricultural ditch in close to Ain El-Balad (Qasr El-Farafra), 3 = Bir 1 (Qasr El-Farafra), 4 = Well of drinking water treatment plant, Qasr El-Farafra (before water treatment), $\mathbf{5}=$ Well of drinking water treatment plant, Qasr El-Farafra (after water treatment), $6=$ Ain El-Hateyya (Qasr El-Farafra), 7 = Ain Bishwa (Qasr El-Farafra), 8 = Bir Gelaw (Qasr ElFarafra), 9 = Ain Goshna (Qasr El-Farafra), 10 = Bir El-Hurra (Qasr El-Farafra), 11= Bir Sitta (Qasr El-Farafra), 12 = Bir El-Sheikh Marzouk (El-Sheikh Marzouk village), 13 = Bir 51 (Abu Hurrira village), $\mathbf{1 4}=$ Bir 1A (Abu Minqar village), $\mathbf{1 5}=$ Bir 1B (Abu Minqar village), $\mathbf{1 6}=$ Bir 9 (Abu Minqar village), $\mathbf{1 7}=$ Well of drinking water treatment plant, Abu Minqar village (before water treatment), $\mathbf{1 8}=$ Well of drinking water treatment plant, Abu Minqar village (after water treatment), $\mathbf{1 9}=$ Bir 7(Lewa Soubah village), 20 = Agricultural drainage (Lewa Soubah village), 21 = Lewa Soubah lake (Lewa Soubah village), 22 = Bir 4 "Bir El-Gazwarin" (El-Nahda village), 23 =Abu Nuss lake (El-Nahda village), 24 = Bir 3A (Esha Abd El-Rahman village).

Sampling: During two meteorologically different successive seasons (summer 2011 and winter 2012), 45 Water samples (representing $21 \& 24$ water samples in summer 2011 and winter 2012, respectively) were collected in clean sterilized 1-L 
polyethylene bottles for determination of the physico-hydrochemical variables of 24 distinct sites (Map 1), where the stations 2, 4 \& 17 were only analyzed chemically in winter 2012. Collection sites were mainly concentrated on the ambient-to-thermal and slightly hot springs and drilled wells, with little attention to other ecosystems (two Drinking Water Treatment Plants "DWTPs", two lakes, an agricultural water-fed ditch and drainage). Sampling sites were not chosen randomly, but were selectively nominated on the basis of likelihood of harboring interesting algal taxa. Not sampled other resources were not accessible at collection time. Water temperature $\left({ }^{\circ} \mathrm{C}\right), \mathrm{pH}$, electrical conductivity $\left(\mu \mathrm{S} . \mathrm{cm}^{-1}\right)$, Total Dissolved Solids (T.D.S.) (mg $\left.\mathrm{L}^{-1}\right)$ were measured in situ with portable Temp./pH/EC/T.D.S. meter (HANNA HI991301) calibrated in the field.

59 different algal samples were collected from all these different watercourses. The algal materials were collected in sterile clean polyethylene bottles according to Bellinger and Sigee (2010). Colored benthic algal communities were collected by hand in sterile polyethylene bottles (Cantonati et al., 2007b). The fresh materials were transported chilled in ice box to the laboratory and then examined, and finally preserved by adding $4 \%$ (v/v) formaldehyde solution.

\section{Laboratory Methods:}

\section{Hydrochemical characteristics}

Water samples were firstly filtered through $0.45 \mu \mathrm{m}$ sterile cellulose acetate membrane filters (Whattman ${ }^{\circledR} \mathrm{GF} / \mathrm{C}$ ) to get rid of plant debris and large soil particles, and then were analyzed to determine the major ions according to the methods adopted by Chapman and Pratt (1978) for $\mathrm{Na}^{+}, \mathrm{K}^{+}, \mathrm{Ca}^{2+}, \mathrm{Mg}^{2+}, \mathrm{Cl}^{-}$, $\mathrm{SO}_{4}{ }^{2-}, \mathrm{CO}_{3}{ }^{2-} \& \mathrm{HCO}_{3}{ }^{-}$, and APHA (2005) for nitrite $\left(\mathrm{NO}_{2}-\mathrm{N}\right)$, nitrate $\left(\mathrm{NO}_{3}-\mathrm{N}\right)$, Soluble Reactive Phosphorus (SRP) and silicate as $\mathrm{SiO}_{2}$. Heavy metals $(\mathrm{Fe}, \mathrm{Cu}$, $\mathrm{Zn} \& \mathrm{Mn}$ ) were also analyzed in terms of Chapman and Pratt (1978). Water hardness as $\mathrm{CaCO}_{3}$ was calculated from the concentration of $\mathrm{Ca}^{2+}$ and $\mathrm{Mg}^{2+}$ ions (APHA, 2005) and typed following Sawyer and McCarty's (1967) 4-pointed scales (soft water: $0-75$, moderately hard water: $75-150$, hard water: $150-300$, very hard water: over $300 \mathrm{mg} \mathrm{L}^{-1} \mathrm{CaCO}_{3}$ ). The classification system of groundwater (Carroll, 1962) was followed to classify these watercourses (freshwater: 0-1000, brackish water: $1000-10000$, saline water: $10000-100000 \mathrm{mg} \mathrm{L}^{-1}$ T.D.S.).

\section{Media and culture conditions}

One $\mathrm{ml}$ of each different collected water sample was inoculated in $50 \mathrm{ml}$ different sterilized liquid growth media: Kolkwitz's solution (Kolkwitz, 1922), Chu \#10, BBM and modified BG-11 media (Andersen et al., 2005) in $100 \mathrm{ml}$ Erlenmeyer flasks in triplicates. The inoculums-containing cultures were maintained under $16 / 8 \mathrm{~h} \mathrm{~L} / \mathrm{D}$ cycle at about $55 \mu \mathrm{mol} \mathrm{m} \mathrm{m}^{-2} \mathrm{~s}^{-1}$ of $20-\mathrm{W}$ cool white 
fluorescent lamps with a temperature of $24 \pm 1{ }^{\circ} \mathrm{C}$. The flasks were usually shaken daily and the algal growths were usually appeared after 2-3 weeks from inoculation date. To isolate unialgal and axenic strains, subsequent continuous culturing on sterilized agarized solid media was performed (Guillard, 2005). The voucher specimens and algal isolates are deposited in Phycology Unit (No. 341), Botany Department, Faculty of Science at Ain Shams University, Cairo, Egypt.

\section{Algal Identification}

The preserved algal materials were identified by light microscopy (LM) and had photomicrographed by $\mathrm{BEL}^{\circledR}$ photonics biological microscope fitted with a Canon Powershot G12 digital camera. The Plate containing (LM) photomicrographs were digitally manipulated and mounted using Adobe Photoshop (version CS 4, Adobe Systems Inc.). The classification systems of Zabelina et al. (1951), Gollerbach et al. (1953), De Desenko-Schegolova et al. (1959), Desikachary, (1959), Randhawa (1959), De Desenko-Schegolova and Gollerbach (1962), Philipose (1967), West and West (1971a, b \& c), Komárek and Anagnostidis (2005) and Wolowski and Hindák (2005) were adopted for algal identification.

\section{Statistical analysis}

Soyer's (1970) frequency index $(F)$ of a particular species $[\mathbf{F}=\mathbf{m} / \mathbf{M} \times \mathbf{1 0 0}$, where $\mathrm{m}=$ number of stations where the species was found; $\mathrm{M}=$ number of all stations, was applied with a simple modification of adding $\mathrm{F}$ value $=0$ (means absent), to determine the frequencies of species in the different localities throughout the whole investigated two periods. Results were evaluated as constant ( $\mathrm{F} \geq 50 \%)$, common $(50 \%>\mathrm{F} \geq 25 \%)$ and rare $(\mathrm{F}<25 \%)$.

Bray-Curtis dissimilarity indices of naturally-propagated and cultured algal assemblages among the different stations were measured based on the farthest neighbor, constrained clustering strategy by using Multi-Variate Statistical Analysis Package (MVSP) version 3.12b provided by Kovach (2000), Kovach computer Service, UK. Also, the Canonical Corresponding Analysis (CCA) was adopted to assess the relationships between the variable environmental parameters and the recorded algal divisions by using the same program. 


\section{Results}

\section{Hydrochemical characteristics and mineral composition:}

Hydrochemical characteristics of all studied habitats are summarized in Table 1. They simply reflect hydrochemistry and lithology of Nubian Sandstone Aquifer System in El-Farafra Oasis as the only main groundwater resource in this isolated desert habitat. In general, most localities have sodium-chloride $\mathrm{NaCl}$-water type, with relevant amounts of $\mathrm{Ca}\left(\mathrm{HCO}_{3}\right)_{2^{-}}, \mathrm{CaCl}_{2^{-}}, \mathrm{Mg}\left(\mathrm{HCO}_{3}\right)_{2^{-}}$and $\mathrm{MgCl}_{2}$ salts (Table 1).

Ain El-Balad slightly hot spring $\left(38^{\circ} \mathrm{C}\right)$ is only the lively still pumping spring (evident flow without turbulent), while other ambient-to-thermal springs are with still stagnant water. Water temperature of Ain El-Bald (station 1) was markedly constant during both studied seasons $\left(38{ }^{\circ} \mathrm{C}\right)$, whilst there was a noticeable fluctuation in water temperature in range of $10{ }^{\circ} \mathrm{C}$ at Ain Bishwa $\left(28{ }^{\circ} \mathrm{C}\right.$ in summer 2011 and $18{ }^{\circ} \mathrm{C}$ in winter 2012). Drilled wells have temperature gradients of $25-42{ }^{\circ} \mathrm{C}$ in summer season 2011 to $32-49{ }^{\circ} \mathrm{C}$ in winter season 2012 . Thus, they are classified as thermal to hot ecosystems (see Glazier's classification, 2009). The two lakes and agricultural water-fed ditch and drainage had a fluctuated water temperature range of $14^{\circ} \mathrm{C}$. In winter season 2012, the lowest water temperature of $14{ }^{\circ} \mathrm{C}$ was recorded at the agricultural drainage (station 20), while the highest degree was $28{ }^{\circ} \mathrm{C}$ recorded at both lakes in summer 2011. Expectedly, pH of springs and wells ranged between 6.47 and 8.43, while other water ecosystems had alkaline nature (Table 1). Electric conductivity (E.C.) was mainly related to the lithology of Nubian Sandstone Aquifer in this desert area and mostly with minor fluctuations in the majority of springs and wells. Ain El-Balad, Ain ElHateyya and Ain Bishwa are mainly freshwater springs with average E.C. values of 219-953 $\mu \mathrm{S} . \mathrm{cm}^{-1}$, while Ain Goshna (station 9) was the only brackish-saline spring with EC of 5650 and $15290 \mu \mathrm{S} . \mathrm{cm}^{-1}$ in summer 2011 and winter 2012, respectively. E.C. values of all drilled wells throughout the whole studied period were low to moderate with freshwater type $\left(173.7-756 \mu \mathrm{S} . \mathrm{cm}^{-1}\right)$. Water hardness of most springs and drilled wells (Table 1) were soft to moderately hard, except Ain Goshna was very hard. Lakes and agricultural water-fed ditch and drainage comprised hard to very hard water types, because of water drainage from agricultural salty lands. With respect to cations and anions concentrations, only Ain Goshna obviously embraced high concentrations of $\mathrm{Ca}^{2+}, \mathrm{Na}^{+}, \mathrm{K}^{+}$and $\mathrm{Mg}^{2+}$ of 3563.28, 701.87, 399.33 and $178.54 \mathrm{mg} \mathrm{L}^{-1}$, respectively and 2011.61-3824.19 $\mathrm{mg} \mathrm{L}^{-1}$ for $\mathrm{Cl}^{-}, 241.49-1062.27 \mathrm{mg} \mathrm{L}^{-1}$ for $\mathrm{SO}_{4}{ }^{2-}$ and $146.25-3824.19 \mathrm{mg} \mathrm{L}^{-1}$ for bicarbonate. All other drilled wells have approximately normal balanced ions concentrations. Nitrate and SRP concentrations in springs and drilled wells were commonly higher than expected for these isolated desert-dwelling habitats (Table 1). Silicates concentrations were low in most sites. Finally, heavy metals values, 
specifically those of $\mathrm{Fe}, \mathrm{Mn}$, followed by little amounts of $\mathrm{Zn}$ were obviously high in most sites.

\section{Algal communities:}

Overall, 82 algal taxa representing 6 algal divisions (cyanoprokaryotes, diatoms, xanthophytes, euglenoids, chlorophytes and charophytes) were recorded from both naturally-propagated (mainly benthic) materials and water-cultivated samples (Tables 2 \& 3, Figs 2-52). In total, 14 taxa that had only been appeared in applying culturing conditions: Amorphonostoc punctiforme f. populorum, Aphanothece microscopica, Cyanosarcina chroococcoides, Phormidium terebriforme, Synechococcus aeruginosus and Synechocystis pevalekii as cyanoprokaryotes; Chloridella simplex, Heterothrix ulotrichoides, Monodus dactylococcoides and Pleurochloris pyrenoidosa representing xanthophytes; Chlorella vulgaris, Scenedesmus bijugatus, S. obliquus and S. quadricauda var. eualternans belonging to chlorophyta. They were completely absent in naturallypropagated materials.

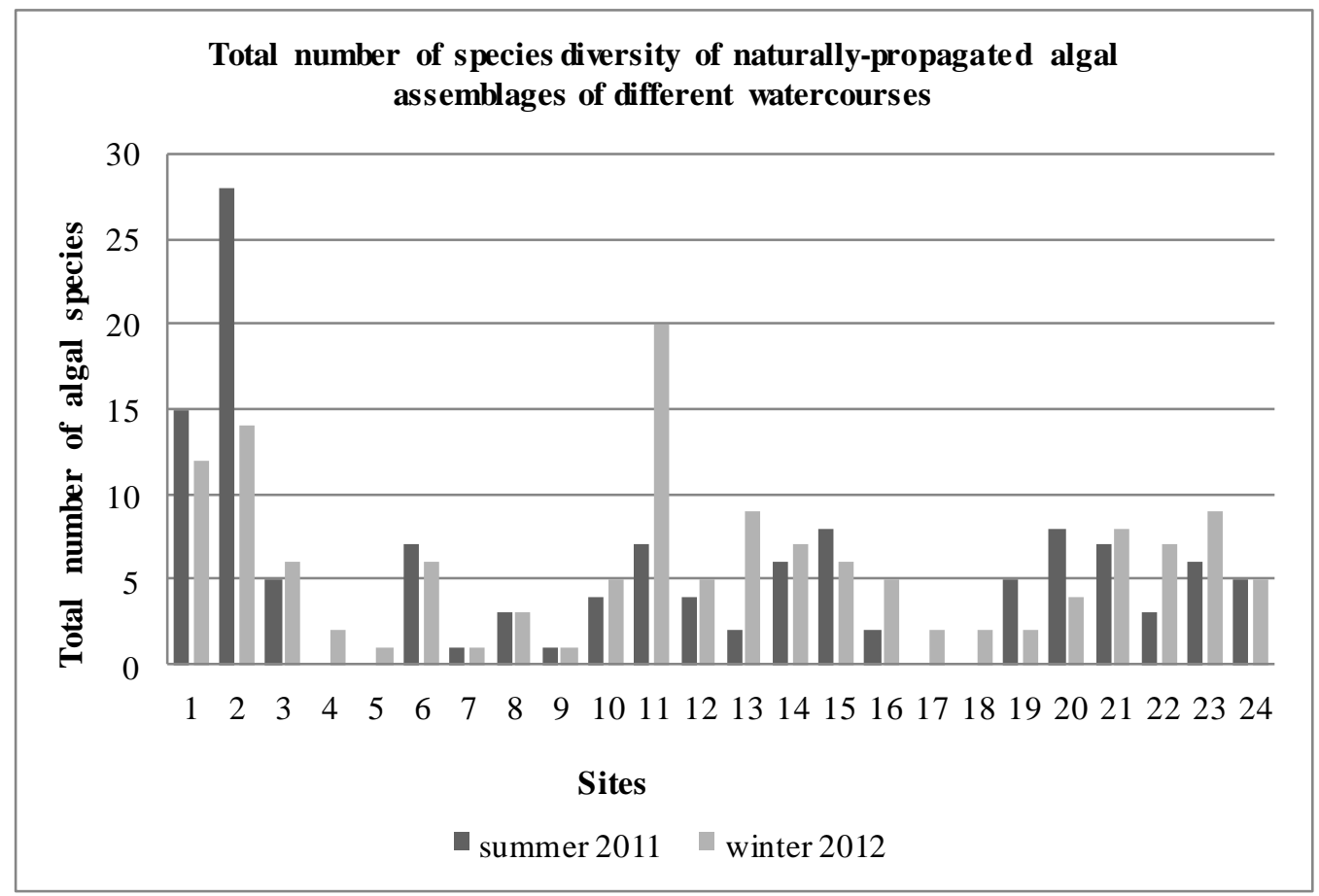

Fig 1. Relationship between the total numbers of naturally-propagated, recorded algal taxa in each of different watercourses of El-Farafra Oasis in this study. 
Table 1. Min-maximum range of hydrochemical characteristics of different watercourses in this study.

\begin{tabular}{|c|c|c|c|c|c|c|c|c|c|}
\hline \multirow[b]{2}{*}{$\begin{array}{l}\text { Parameters } \\
\text { Pats }\end{array}$} & \multirow[b]{2}{*}{$\begin{array}{c}\text { Unit } \\
\end{array}$} & \multicolumn{2}{|c|}{ Springs (Ains) } & \multicolumn{2}{|c|}{ Wells (Birs) } & \multicolumn{2}{|c|}{ Lakes } & \multicolumn{2}{|c|}{ Agricultural ditch \& drainage } \\
\hline & & summer2011 & Winter 2012 & Summer 2011 & Winter 2012 & summer 2011 & winter 2012 & $\begin{array}{c}\text { summer } \\
2011\end{array}$ & $\begin{array}{l}\text { winter2012 } \\
\text { wat }\end{array}$ \\
\hline Temp. & ${ }^{\circ} \mathrm{C}$ & $24-38$ & $18-38$ & $25-42$ & $32-49$ & 28 & $15-17$ & 24 & $14-20$ \\
\hline pH & & $7.44-8.43$ & $7.47-7.77$ & $6.50-7.53$ & $6.47-7.76$ & $7.6-8.13$ & $7.50-7.71$ & 7.72 & $7.25-8.63$ \\
\hline E.C. & $\mu \mathrm{S} . \mathrm{cm}^{-1}$ & $288-5650$ & $219-15290$ & $173.7-756$ & $191-756$ & $3620-27300$ & $654-2320$ & 7760 & $296-2190$ \\
\hline \multirow[t]{2}{*}{ T.D.S. } & $\mathrm{mg} \mathrm{L}^{-1}$ & $200-3950$ & $220-10100$ & $110-500$ & $130-560$ & $2520-28000$ & $450-1590$ & 5420 & $200-1510$ \\
\hline & Type & Fresh - Brackish & Fresh - Saline & Fresh & Fresh & Brackish - Saline & Fresh - Brackish & Brackish & Fresh - Brackish \\
\hline \multirow{2}{*}{$\begin{array}{l}\text { Hardness (as } \\
\mathrm{CaCO}_{3} \text { ) }\end{array}$} & $\mathrm{mg} \mathrm{L}^{-1}$ & $77.91-1557.87$ & $52.99-8947.05$ & $15.72-177.95$ & $42.69-177.19$ & $554.53-4094.84$ & $178.99-804.03$ & 2444.11 & $81.42-397.44$ \\
\hline & Type & moderately hard - very hard & soft - very hard & soft - hard & soft - hard & very hard & hard - very hard & very hard & moderately hard - very hard \\
\hline $\mathrm{Na}^{+}$ & $\mathrm{mg} \mathrm{L}^{-1}$ & $18.92-701.87$ & $20.10-138.19$ & $2.20-78.47$ & $10.25-76.97$ & $565.78-6067.25$ & $54.15-160.32$ & 884.76 & $20.38-211.83$ \\
\hline $\mathbf{K}^{+}$ & $\mathrm{mg} \mathrm{L}^{-1}$ & $19.56-143.24$ & $10.05-399.33$ & $1.76-41.40$ & $3.41-50.52$ & $19.94-795.40$ & $6.26-23.77$ & 93.48 & $17.47-185.35$ \\
\hline $\mathrm{Ca}^{2+}$ & $\mathrm{mg} \mathrm{L}^{-1}$ & $14.21-329.45$ & $9.95-3563.28$ & $0.23-38.43$ & $4.4-54.98$ & $161.65-1183.85$ & $60.09-293.27$ & 667.74 & $18.92-117.26$ \\
\hline $\mathrm{Mg}^{2+}$ & $\mathrm{mg} \mathrm{L}^{-1}$ & $7.19-178.54$ & $4.58-15.25$ & $1.14-19.91$ & $2.72-11.1$ & $36.64-276.53$ & $7.03-17.42$ & 188.62 & $8.30-25.41$ \\
\hline $\mathrm{Cl}^{-}$ & $\mathrm{mg} \mathrm{L}^{-1}$ & $20.93-2011.61$ & $48.44-3824.19$ & $26.65-200.24$ & $42.36-182.71$ & $1020.58-12859.77$ & $92.25-487.94$ & 3098.34 & $72.51-564.27$ \\
\hline $\mathrm{SO}_{4}^{2-}$ & $\mathrm{mg} \mathrm{L}^{-1}$ & $0-241.49$ & $16.14-1062.27$ & $0-30.55$ & $6.47-65.36$ & $58.93-260.07$ & $101.87-155.53$ & 1.80 & $14.30-93.32$ \\
\hline $\mathrm{HCO}_{3}$ & $\mathrm{mg} \mathrm{L}^{-1}$ & $8.48-146.25$ & $29.78-3824.19$ & $15.77-93.97$ & $19.35-111.63$ & $70.76-89.59$ & $110.96-378.35$ & 103.49 & $37.67-246.74$ \\
\hline $\mathrm{CO}_{3}{ }^{2-}$ & $\mathrm{mg} \mathrm{L}^{-1}$ & $3.65-34.98$ & 0.0 & $0-18.13$ & 0.0 & $16.24-31.88$ & 0.0 & 17.95 & 0.0 \\
\hline $\mathrm{NO}_{3}-\mathrm{N}$ & $\mu \mathrm{g} \mathrm{L}^{-1}$ & $60-1410$ & $73-1106$ & $40-520$ & $56-154$ & $1800-5400$ & $57-7500$ & 3600 & $55-7500$ \\
\hline $\mathrm{NO}_{2}-\mathrm{N}$ & $\mu \mathrm{g} \mathrm{L}^{-1}$ & $0-60$ & $0-4$ & 0.0 & 0.0 & $70-120$ & $26-60$ & 200 & $0-24$ \\
\hline SRP & $\mu \mathrm{g} \mathrm{L}^{-1}$ & $296-382$ & $16-44$ & $243-420$ & $0-67$ & $38-368$ & $0-7$ & 320 & $0-25$ \\
\hline Silicate as $\mathrm{SiO}_{2}$ & $\mathrm{mg} \mathrm{L}^{-1}$ & $2.33-5.40$ & $1.38-7.40$ & $0.89-5.84$ & $1.47-4.95$ & $3.02-3.13$ & $1.11-2.53$ & 5.04 & $1.60-2.73$ \\
\hline Fe & $\mu \mathrm{g} \mathrm{L}^{-1}$ & $760-1190$ & $220-430$ & $540-1120$ & $111-380$ & $1080-1240$ & $220-270$ & 860 & $270-380$ \\
\hline Mn & $\mu \mathrm{g} \mathrm{L}^{-1}$ & $40-6040$ & $220-380$ & $0-580$ & $200-680$ & $140-160$ & $260-300$ & 40 & $0-320$ \\
\hline Zn & $\mu \mathrm{g} \mathrm{L}^{-1}$ & $110-170$ & $180-460$ & $100-200$ & $160-1080$ & $180-260$ & $200-260$ & 130 & $140-200$ \\
\hline $\mathrm{Cu}$ & $\mu \mathrm{g} \mathrm{L}^{-1}$ & $0-30$ & $30-120$ & $0-30$ & $0-60$ & 10 & 30 & 20 & $0-30$ \\
\hline
\end{tabular}


Table 2. Naturally-propagated algal assemblages of different watercourses (drilled wells, ambient-to-thermal and slightly hot springs, DWTPs, agricultural ditch \& drainage, and lakes) in this study.

\begin{tabular}{|c|c|c|c|c|c|c|c|c|c|c|c|c|c|c|c|c|c|c|c|c|c|c|c|c|c|}
\hline & Seasons & 1 & 2 & 3 & 4 & 5 & 6 & 7 & 8 & 9 & 10 & 11 & 12 & 13 & 14 & 15 & 16 & 17 & 18 & 19 & 20 & 21 & 22 & 23 & 24 \\
\hline \multirow{2}{*}{ Cyanoprokaryota } & summer 2011 & 14 & 15 & 5 & - & - & 7 & 1 & 3 & 1 & 4 & 7 & 4 & 2 & 6 & 8 & 2 & - & - & 5 & 3 & 4 & 3 & 4 & 4 \\
\hline & winter 2012 & 9 & 3 & 6 & 2 & 1 & 6 & 1 & 3 & 1 & 5 & 15 & 5 & 9 & 5 & 6 & 5 & 2 & 2 & 2 & 2 & 4 & 6 & 6 & 4 \\
\hline \multirow{2}{*}{ Bacillariophyta } & summer 2011 & 1 & 3 & - & - & - & - & - & - & - & - & - & - & - & - & - & - & - & - & - & - & - & - & - & - \\
\hline & winter 2012 & 3 & 3 & - & - & - & - & - & - & - & - & - & - & - & - & - & - & - & - & - & - & - & - & - & - \\
\hline \multirow{2}{*}{ Xanthophyta } & summer 2011 & - & - & - & - & - & - & - & - & - & - & - & - & - & - & - & - & - & - & - & - & - & - & - & - \\
\hline & winter 2012 & - & 3 & - & - & - & - & - & - & - & - & - & - & - & - & - & - & - & - & - & - & - & - & - & - \\
\hline \multirow{2}{*}{ Euglenophyta } & summer 2011 & - & - & - & - & - & - & - & - & - & - & - & - & - & - & - & - & - & - & - & 4 & 2 & - & - & - \\
\hline & winter 2012 & - & - & - & - & - & - & - & - & - & - & - & - & - & - & - & - & - & - & - & - & 2 & - & 2 & - \\
\hline \multirow{2}{*}{ Chlorophyta } & summer 2011 & - & 10 & - & - & - & - & - & - & - & - & - & - & - & - & - & - & - & - & - & 1 & 1 & - & 2 & 1 \\
\hline & winter 2012 & - & 4 & - & - & - & - & - & - & - & - & 5 & - & - & 2 & - & - & - & - & - & 2 & 2 & 1 & 1 & 1 \\
\hline \multirow{2}{*}{ Charophyta } & summer 2011 & - & - & - & - & - & - & - & - & - & - & - & - & - & - & - & - & - & - & - & - & - & - & - & - \\
\hline & winter 2012 & - & 1 & - & - & - & - & - & - & - & - & - & - & - & - & - & - & - & - & - & - & - & - & - & - \\
\hline $\begin{array}{l}\text { Total number of } \\
\text { all recorded algal } \\
\text { taxa }\end{array}$ & & & & & & & & & & & & & 68 & & & & & & & & & & & & \\
\hline
\end{tabular}


Table 3. Summary of cultivated algal taxa of different water samples on all applied culture media (Kolkwitz's solution, Chu \#10 medium, BBM \& Mod. BG-11 media) in this study.

\begin{tabular}{|c|c|c|c|c|c|c|c|c|}
\hline \multirow{3}{*}{$\begin{array}{l}\text { Algal Taxa } \downarrow \\
\text { Division: Cyanophyta }\end{array}$} & \multicolumn{2}{|c|}{ Kolkwitz's solution } & \multicolumn{2}{|c|}{$\begin{array}{l}\text { Chu \#10 } \\
\text { Medium } \\
\end{array}$} & \multicolumn{2}{|c|}{$\begin{array}{c}\text { Bold Basal Medium } \\
\text { (BBM) }\end{array}$} & \multicolumn{2}{|c|}{$\begin{array}{c}\text { Mod. BG-11 } \\
\text { Medium }\end{array}$} \\
\hline & $\begin{array}{c}\text { Summer } \\
2011\end{array}$ & $\begin{array}{l}\text { Winter } \\
2012\end{array}$ & $\begin{array}{c}\text { Summer } \\
2011\end{array}$ & $\begin{array}{l}\text { Winter } \\
2012\end{array}$ & $\begin{array}{l}\text { Summer } \\
2011\end{array}$ & $\begin{array}{l}\text { Winter } \\
2012\end{array}$ & $\begin{array}{c}\text { Summer } \\
2011\end{array}$ & $\begin{array}{l}\text { Winter } \\
2012\end{array}$ \\
\hline & & & & & & & & \\
\hline Amorphonostoc punctiforme f. populorum (Geitl.) Hollerb. & & & & + & & + & + & + \\
\hline Anabaena constricta (Szafer) Geitl. & & & + & + & & & + & + \\
\hline Aphanothece microscopica Näg. & & & + & & & & + & \\
\hline Calothrix elenkinii Kossinsk. & & & + & + & + & + & + & + \\
\hline Chroococcus minutus (Kütz.) Näg. & & + & + & + & & + & & + \\
\hline C. turgidus (Kütz.) Näg. & & & + & + & & & + & + \\
\hline Cyanosarcina chroococcoides (Geit.) Kovácik & & & & + & & & & + \\
\hline Geitlerinema jasorvense (Vouk) Anagnostidis & + & + & + & + & + & + & + & + \\
\hline Limnothrix redekei (Goor) Meffert & & & + & & + & & + & \\
\hline Oscillatoria tenuis Ag. & + & & & & & & & \\
\hline Phormidium carboniciphilum (Prát) Anagnostidis \& Komárek & + & & + & + & & & + & \\
\hline Phormidium okenii (C.Ag. ex Gom.) Anagnostidis \& Komárek & + & & + & & & & + & \\
\hline Phormidium terebriforme (C.Ag. ex Gom.) Anagnostidis \& Komárek & + & & & & + & & + & + \\
\hline Planktolyngbya limnetica (Lemm.) Komárková-Legnerová \& Cronberg & + & + & + & + & + & + & + & + \\
\hline Sphaeronostoc microscopicum (Carm.) Elenk. & & & + & & & & + & \\
\hline Stratonostoc linckia f. calcicola (Bréb.) Elenk. & & & + & + & & + & & + \\
\hline Synechococcus aeruginosus Näg. & + & & & & & & & \\
\hline Talglęéchøenstisuedditekii Erceg. & & & + & + & & + & + & + \\
\hline Westiellopsis prolifica Janet & & & + & + & + & + & + & + \\
\hline
\end{tabular}




\begin{tabular}{|c|c|c|c|c|c|c|c|c|c|}
\hline \multicolumn{10}{|c|}{ Division: Xanthophyta } \\
\hline \multicolumn{2}{|c|}{ Chloridella simplex Pasch. } & + & + & + & + & & & + & + \\
\hline \multicolumn{2}{|c|}{ Heterothrix ulotrichoides Pasch. } & & & + & & & + & & \\
\hline \multicolumn{2}{|c|}{ Monodus dactylococcoides Pasch. } & & & & + & & & & + \\
\hline \multicolumn{2}{|c|}{ Pleurochloris pyrenoidosa Pasch. } & + & + & + & + & + & + & + & + \\
\hline \multicolumn{10}{|c|}{ Division: Chlorophyta } \\
\hline \multicolumn{2}{|c|}{ Chlamydomonas globosa Snow } & & & + & + & & & & + \\
\hline \multicolumn{2}{|c|}{ Chlorella vulgaris Beijerinck } & + & & & & + & & & \\
\hline \multicolumn{2}{|c|}{ Scenedesmus bijugatus Kütz. } & & & & + & & + & & + \\
\hline \multicolumn{2}{|c|}{ S. obliquus (Turpin) Kütz. } & + & & + & + & & + & & \\
\hline \multicolumn{2}{|c|}{ S. quadricauda (Turpin) Brébisson } & + & & + & + & & + & & + \\
\hline \multicolumn{2}{|c|}{ S. quadricauda var. eualternans Proshkina-Lavrenko } & & & & + & & & & \\
\hline \multirow{2}{*}{ Cyanoprokaryotes } & NO. of taxa & 7 & 3 & 14 & 12 & 6 & 8 & 14 & 12 \\
\hline & Percentage $(\%)$ & 58.33 & 60.0 & 70.0 & 60.0 & $\mathbf{7 5 . 0}$ & 61.54 & 87.50 & 66.67 \\
\hline \multirow{2}{*}{ Xanthophytes } & NO. of taxa & 2 & 2 & 3 & 3 & 1 & 2 & 2 & 3 \\
\hline & Percentage $(\%)$ & 16.67 & 40.0 & 15.0 & 15.0 & 12.50 & 15.38 & 12.50 & 16.67 \\
\hline \multirow{2}{*}{ Chlorophytes } & NO. of taxa & 3 & $\mathbf{0}$ & 3 & 5 & 1 & 3 & $\mathbf{0}$ & 3 \\
\hline & Percentage $(\%)$ & 25.0 & 0.0 & 15.0 & 25.0 & 12.50 & 23.08 & 0.0 & 16.67 \\
\hline \multicolumn{2}{|c|}{ Total number of taxa } & 12 & 5 & 20 & 20 & 8 & 13 & 16 & 18 \\
\hline \multicolumn{3}{|c|}{ Total number of cyanoprokaryote taxa } & & & & 19 & & & \\
\hline \multicolumn{3}{|c|}{ Total number of xanthophycean taxa } & & & & 4 & & & \\
\hline \multicolumn{3}{|c|}{ Total number of chlorophycean taxa } & & & & 6 & & & \\
\hline \multicolumn{3}{|c|}{ Total number of all cultivated algal taxa } & & & & 29 & & & \\
\hline
\end{tabular}




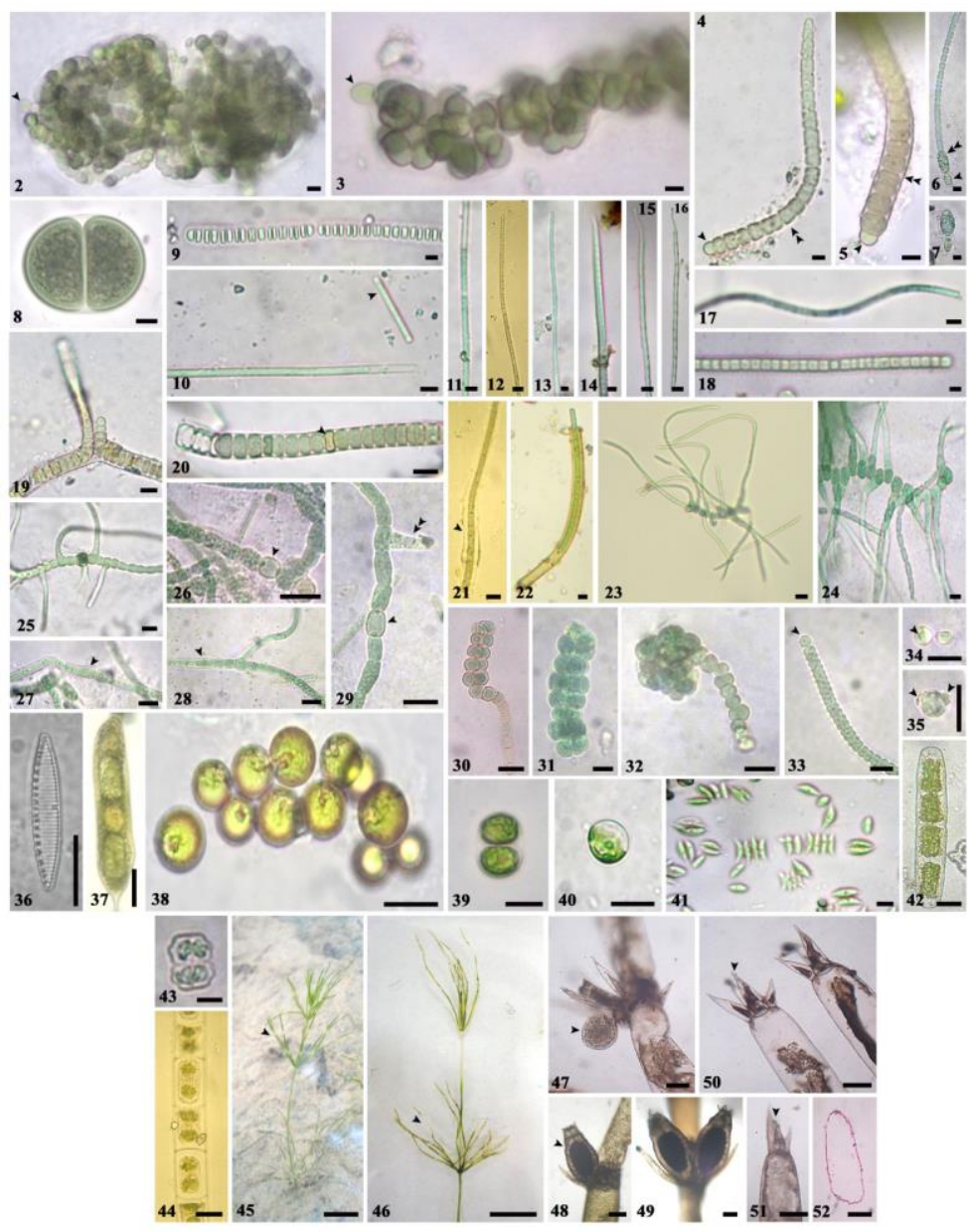

Figs 2-52. Some commonly naturally-propagated and isolated algal strains in El-Farafra Oasis during this study. Scale bar $=5 \mu \mathrm{m}$ (Figs 2-18; 41); Scale bar $=10 \mu \mathrm{m}$ (Figs 19-22; 24-36; 38-40; 42-43); Scale bar $=20 \mu \mathrm{m}$ (Figs 23; 37; 44); Scale bar $=1 \mathrm{~cm}$ (Figs 45-46), Scale bar $=500 \mu \mathrm{m}$ (Fig 47); Scale bar $=200 \mu \mathrm{m}$ (Figs 48-52).

Figs 2-3. Amorphonostoc punctiforme f. populorum with spherical or subspherical heterocysts (arrowheads).

Figs 4-5. Calothrix elenkinii with immature basal heterocyst (arrowhead, Fig. 4) and bent at the base (double arrowheads, Fig. 4); with mature basal heterocyst (arrowhead, Fig. 5) and thin, non-lamellated sheath close to the trichome (double arrowheads, Fig. 5). Figs 6-7. Cylindrospermum alatosporum with ellipsoidal basal heterocyst (arrowhead) and akinete (double arrowheads). Fig. 8. Chroococcus turgidus. Fig. 9. Johannesbaptistia pellucida. Figs 10-11. Planktolyngbya limnetica showing liberated cell (arrowhead, Fig 10). Figs 12-13. Geitlerinema jasorvense. Fig. 14. Phormidium acuminatum. Figs 15-16. Geitlerinema splendidum. Fig. 17. Jaaginema subtilissimum. Fig 18. Oscillatoria tenuis. Figs 19-20. Scytonema ocellatum showing false branching (Fig. 19) and subquadrate heterocyst (arrowhead, Fig. 20). Figs 21-22. Symploca dubia showing outer yellowish firm uneven sheath (arrowhead, Fig 21). Figs 23-35. Complete life stages of Westiellopsis prolifica showing true Tshaped branching (Figs 23-25), heterocysts (arrowheads, Figs 26-29. Double arrowheads in Fig 29 depicting early stage of lateral branch formation), pseudohormocysts (30-32), escaping of gonidia (arrowhead, Fig. 33), free gonidial cells with refractive bodies (arrowhead, Fig. 34), early stage of germinating gonidial cell (arrowheads, Fig. 35). Fig. 36. Nitzschia amphibia. Fig. 37. Euglena oxyuris. Figs 38-39. Chloridella simplex. Fig. 40. Pleurochloris pyrenoidosa. Fig. 41. Scenedesmus obliquus. Fig. 42. Penium margaritaceum. Fig. 43. Euastrum insulare. Fig. 44. Zygnemopsis sp. Figs 45-52. habitus of Nitellopsis obtusa showing branchlets (arrowheads, Figs 45-46); antheridium (Fig. 47); Oogonium (Figs 48-49); terminal pointed conical cells of branchlets (Figs 50-51); eccorticated axial cell (Fig. 52). 


\section{Naturally-propagated algal flora}

Totally, 68 algal taxa were identified and characterized (Table 2, Fig. 1) throughout the whole studied period. 38 taxa $(55.88 \%)$ of these identified taxa were cyanoprokaryotes, followed by chlorophytes (19 taxa, 27.94\%), with little contribution of euglenoids (4 taxa), xanthophytes ( 3 taxa), diatoms (3 taxa), and only unique expression in winter 2012 for charophytes by over mass growth of Nitellopsis obtusa at spring-fed agriculture ditch in-close-to Ain El-Balad slightly hot spring. The most widely frequent cyanoprokaryotes in summer 2011 were Jaaginema subtilissimum, Oscillatoria limosa and O. tenuis, whilst Jaaginema subtilissimum, Oscillatoria subbrevis, $O$. tenuis and Phormidium carboniciphilum, P. corium and P. okenii were the more dominant in winter 2012. For more details, in summer 2011, 50 algal taxa was found in all habitats; represented by 30 taxa (60\%) of cyanoprokaryotes, followed by 13 chlorophyte taxa (26\%), and the reminder was diatoms and euglenoids of 3 and 4 taxa, respectively. Of these recorded taxa, only Oscillatoria tenuis has been recorded as a constant biological pollution indicator ( $2 \%$, Fig. 53), where was recorded in most habitats. In winter 2012, two more algal groups were observed (xanthophytes and charophytes) and were totally absent in the summer season (Table 2). Of the 51 recorded taxa in winter 2012, cyanoprokaryota accounted for the largest proportion of 31 taxa $(60.78 \%)$, followed by 11 chlorophyte taxa (21.57\%), with much smaller proportions of diatoms 3 taxa $(5.88 \%)$, xanthophytes 3 taxa (5.88\%), euglenoids 2 taxa (3.92\%) and the charophyte Nitellopsis obtusa (1.96\%). The macroscopic xanthophyte Vaucheria geminata and $V$. sessilis were only found at the agricultural ditch nearby Ain El-Balad. Also, O. tenuis shared the constant appearance in most localities with richness of $1.96 \%$ (Fig. 54). In general, most recorded algal taxa were rare in their distribution in all studied habitats, while others were remarkably restricted to the springs and drilled wells, e.g. Calothrix elenkinii, Geitlerinema splendidum, Limnothrix redekei, Scytonema ocellatum and Symploca dubia (check Figs. 2-52).

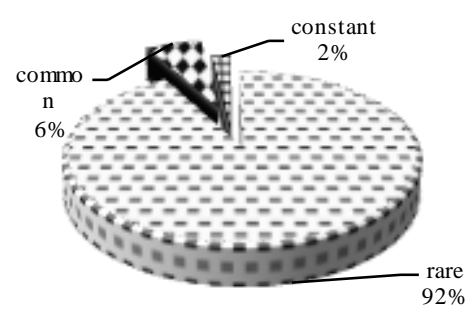

Fig. 53. Distribution of naturally-propagated, recorded algal taxa of all different watercourses based on frequency indices in summer season 2011.

Egyptian J. of Phycol. Vol. 16, 2015

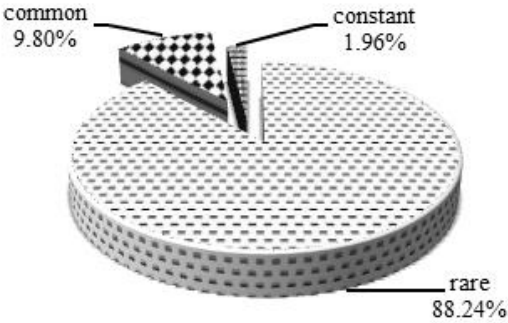

Fig. 54. Distribution of naturallypropagated, recorded algal taxa of all different watercourses based on frequency indices in winter season 2012. 
Bray-Curtis dissimilarity dendogram of all studied sites based on presence/absence of algal species composition in summer 2011 (Fig. 55) revealed that Ain El-Balad slightly hot spring (station I) and its neighbor agriculturalwater-fed ditch (station 2) was distinctly considered as out-group with the highest dissimilarity percentage (67.4\%). Other stations were remarkably separated into two autonomous groups: the first one includes all springs and most of drilled wells, while the other one comprised the two lakes, an agricultural drainage and only two wells (station 22: Bir 4 and station 24: Bir 3A) that might be due to their geographically closer proximity. Noteworthy, there were a complete disappearance of algal taxa in tanks of drinking water treatment plants (DWTPs) before and after water treatment at both stations 4 and 5 (Qasr El-Farafra), and stations 17 and 18 (Abu Minqar village), where both had been cleaned before sampling (Saber, personal communication). In winter 2012, Bray-Curtis dissimilarity ordination was distinctly different (Fig. 56). The highest algal dissimilarity percentage $(85.7 \%)$ was logically between Abu Nuss lake (station 23) and Bir 3A (station 24). Tanks of the DWTPs before and after treatment (stations $17 \& 18$, respectively) at Abu Minqar village were identical from algal structure (with $100 \%$ similarity percentage), where only scum growth of Oscillatoria tenuis was recorded inside these tanks.

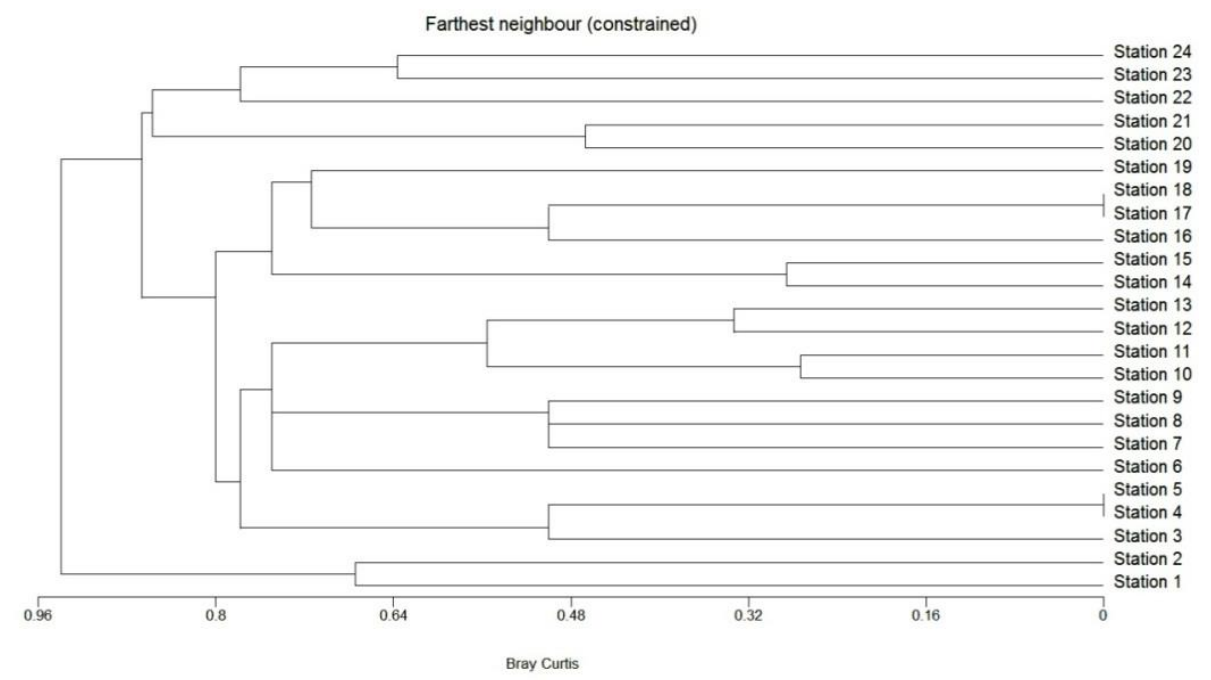

Fig. 55. Bray-Curtis dendogram displaying the dissimilarity relationships among the stations based on presence /absence of naturally-propagated species composition of algal assemblages in summer season 2011. 


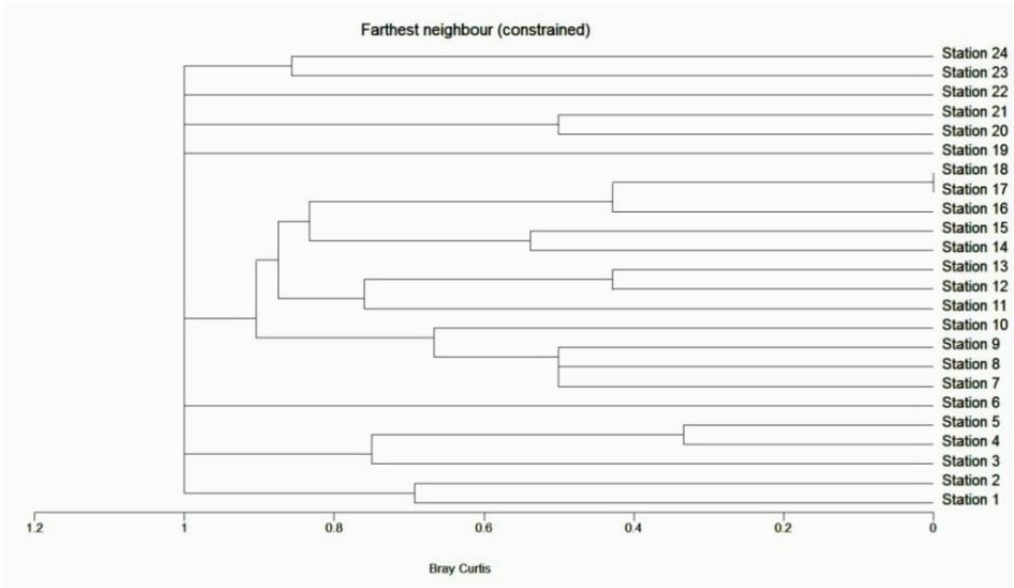

Fig. 56. Bray-Curtis dendogram displaying the dissimilarity relationships among the stations based on presence /absence of naturally-propagated species composition of algal assemblages in winter season 2012.

Canonical Correspondence Analysis (Fig. 57) pointed out a significantly positive correlation between the temperature and cyanoprokaryotes. However, this algal division is inversely correlated with $\mathrm{NO}_{3}-\mathrm{N}, \mathrm{NO}_{2}-\mathrm{N}$ and SRP values. There is also a positive relationship between euglenoids and $\mathrm{NO}_{3}-\mathrm{N}$ and $\mathrm{NO}_{2}-\mathrm{N}$ concentrations, and a remarkable likelihood between chlorophyta and $\mathrm{pH}$.

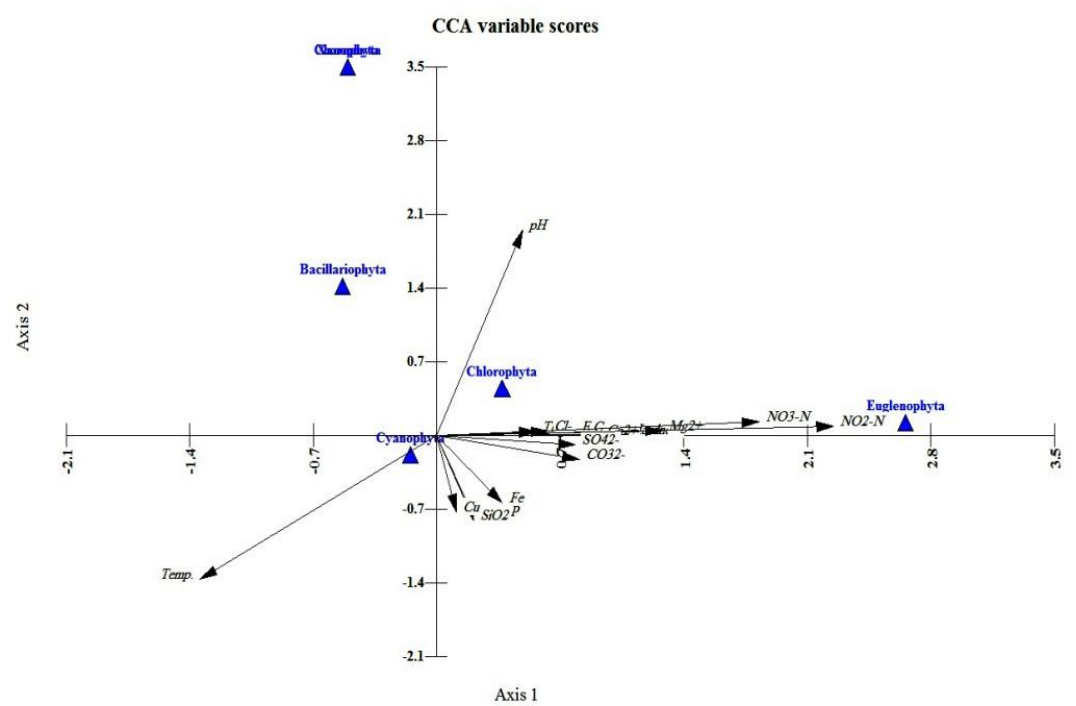

Fig. 57. Canonical Correspondence Analysis (CCA) depicting relationships among naturally-propagated algal communities based on species number in each algal division and physicochemical parameters of all the stations. 


\section{Cultured materials}

Cultured samples revealed three dominant algal divisions (Table 3). In all cultures, 29 species were identified, of which 19 cyanoprokaryotes $(65.52 \%$ of total taxa) represented the highest richness, with little assistance of both 4 chlorophyte taxa $(20.69 \%)$ and 4 xanthophyte taxa (13.79\%). Chu\# 10 medium, followed by modified BG-11 medium harbored the highest abundance of algal taxa in both studied seasons. But, Kolkwitz's solution had an apparently weakened propagation compared to other media, especially in winter season 2012 (only 5 taxa). Cyanoprokaryotes fairly represent the most dominant and frequent division using these culturing techniques. Their highest numbers were 14 taxa recorded in summer season on both Chu\#10 and modified BG-11 media, while the lowest proportion (only 3 taxa) was present in winter season using Kolkwitz's solution (Table 3). Based on frequency indices (Fig. 58), 16 taxa were mainly constant (55.17\% of total taxa) in studied habitats and very much indeed dominated by Calothrix elenkinii, Geitlerinema jasorvense, Planktolyngbya limnetica, Westiellopsis prolifica, Chloridella simplex and Pleurochloris pyrenoidosa. By these culturing techniques, different 12 algal strains had been isolated Amorphonostoc punctiforme f. populorum, Calothrix elenkinii, Geitlerinema jasorvense, Phormidium carboniciphilum, Planktolyngbya limnetica, Stratonostoc linckia f. calcicola, Westiellopsis prolifica, Chloridella simplex, Pleurochloris pyrenoidosa, Chlorella vulgaris, Scenedesmus bijugatus and S. obliquus.

Bray-Curtis dissimilarity clustering (Fig. 59) had splitted the studied localities into two groups based on presence /absence of their cultivated algal taxa. The first one includes the first five biogeographically neighboring stations (1-5). Although the agricultural water-fed ditch was included in this cluster, but its water is mainly spring-fed by Ain El-Bald slightly hot spring (station 1). The second cluster involves the other stations. The lowest algal dissimilarity (5.9\%) was found between drinking water plants before and after treatment (stations $17 \& 18$, respectively) at Abu Minqar village. The highest dissimilarity (47.8\%) was reported between brackish-saline ambient-to-thermal spring Ain Goshna (station 9) and fairly freshwater Bir El-Hurra (station 10).

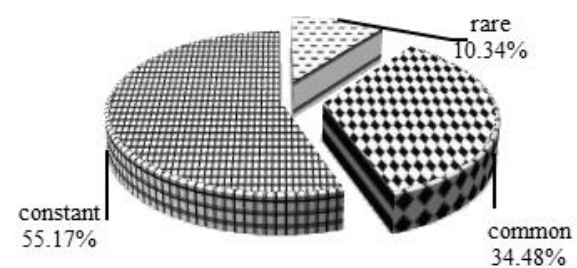

Fig. 58. Frequency scales of cultivated algal taxa on different media during the whole period of study. 


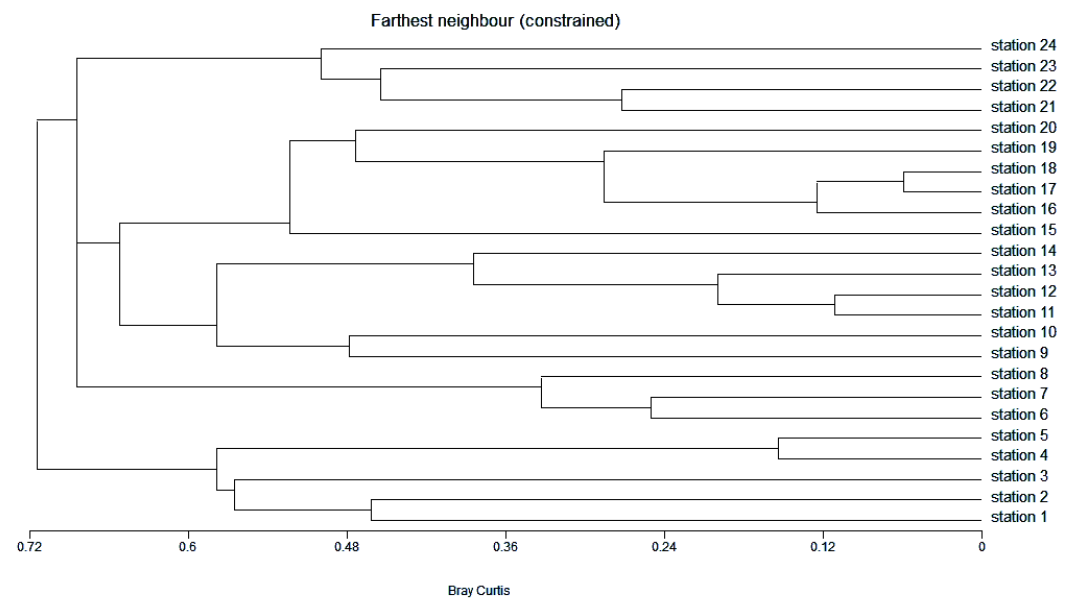

Fig 59. Dendogram of Bray-Curtis dissimilarity showing the relationships among the stations based on presence /absence of species composition of algal assemblages of cultured water inoculums on all four growth media during the whole studied period.

\section{Discussion}

In this study, hydrochemical characterizations of El-Farafra Oasis watercourses, specifically in springs and wells, are the main ecological drivers determining algal species distribution and community composition. This is completely in an agreement with the results of Scarsbrook $\boldsymbol{e t}$ al. (2007) and Cantonati et al. (2012c) in their intensive studies on springs-inhabiting algae. In our work, the min-maximum values of each parameter of the same water set were introduced throughout the investigated period. For springs: Ain El-Balad is the only slightly hot spring with stable temperature of $38^{\circ} \mathrm{C}$, while others are ambient to thermal springs (Glazier, 2009). There was a proximately remarkable stability in the physicochemical properties at Ain El-Balad, while other springs had a bit more fluctuations. Ain Goshna was the most fluctuant spring in its hydrological features and at the same time is the only separated brackish-saline spring in this study. All drilled wells shared freshwater type with slight seasonal variations in their mineralization contents. In congruence with this conclusion, most spring habitats and other similar water systems "wells", compared to other aquatic ecosystems, have greater stable hydrological characterization with little seasonal fluctuations (Williams, 1991a; van der Kamp, 1995; Cantonati et al., 2006). However, this feature varies among spring classes (Scarsbrook et al., 2007). Most springs and wells of El-Farafra Oasis have more or less similar physicochemical features compared to those inhabiting other closer oases in the Western Desert (e.g., Shaaban $\boldsymbol{e t}$ al., 1997 on his study at El-Bahariya Oasis). The hydrochemical characterizations of springs and drilled wells of El-Farafra Oasis merely pointed out that most of them are still bearing partially near-natural 
conditions, with some agricultural, pastures and humans impacts, and with remarkable mineralization patterns, which are coherent with the lithological substrate (Ali, 2002; El Sabri and El Sheikh, 2009). This picture of pollution was strongly expressed from relatively high values of nitrate, sometimes nitrite, and SRP at some springs and drilled wells, where they are highly utilized as pivotal water resources for drinking, agriculture, and cattle breeding. In harmony with our results, contribution of Cantonati et al. (1996) on mountain springs of the River Sarca catchment (Northern Italy). Also, Poulíčková et al. (2003) and Scarsbrook et al. (2007) interestingly denoted to degradation and reduction of springs might be attributed to direct human impacts, or indirect influence due to runoff nutrients deposition. We also concluded that water extraction for municipal and irrigation uses from springs, in addition to normal /or mechanically-pumping drilled wells, had greatly reduced the density of springs in El-Farafra Oasis. Erman (2002) reported that Land drainage of springs for agricultural activities in the Funeral Mountains, Death Valley (USA) has reduced the springs to approximately $15 \%$. In the lakes and agricultural water-fed ditch and drainage, eutrophication phenomenon is high due to water run-off and nutrients-leaching in these habitats, especially in summer season, where more waters had been evaporated and the nutrients concentrated and deposited in these hyper-ariddesert-dwelling ecosystems.

This study also revealed that drinking water treatment plants (DWTPs) in ElFarafra Oasis and hydrochemical composition of some springs and drilled wells are considered just about excellent potable water resources in the context of water quality guidelines of Egyptian Higher Committee for Water (EHCW) and World Health Organization (WHO), but they substantially still need special governmental efforts in the future to mainly eliminate the relatively high contents of heavy metals, especially Fe, Mn and Zn. Ali (2002) reported high presence of these heavy metals, especially Fe, in all studied wells. In general, the DWTPs are required to guarantee the national and WHO water quality standards and have to be fully free of algae. In Egypt, little synchronous hydrochemical and biological attention had been paid to assess drinking water quality (e.g. El-Sebaie $\boldsymbol{e t} \boldsymbol{a l}$, 2002; Khalil $\boldsymbol{e t}$ al., 2013) and how to be treated (Bedir, 2014). Khalil $\boldsymbol{e t}$ al. (2013), on their recent study on water quality in Tanta City, reported that relatively high concentrations of $\mathrm{Fe}, \mathrm{Mn}$ and hardness are markedly exceeding both the WHO and EHCW limits. Interestingly, this evidence confirms our presented results. We think that the more primitive and simplest applied method of water treatment in El-Farafra Oasis has to be urgently modified and updated in the future to reach drinking water standard levels.

In total, 82 algal taxa have been identified in all studied habitats. This number of algal diversity is almost considered relatively high, compared to other published surveying data belong to other neighboring oases in the Western Desert: 69 taxa in Siwa Oasis (Shaaban, 1985), 110 taxa of six thermal springs in ElEgyptian J. of Phycol. Vol. 16, 2015 - 65 - 
Bahariya Oasis (Shaaban et al., 1997), and finally in El-Kharga Oasis, 26 taxa mainly restricted to three wells (Shaaban and El Habibi, 1978) and 233 taxa belonging to wells, agricultural drainage, rice fields and sewage oxidation pond (Gharib, 2004). Most of the identified algal taxa harboring the springs and wells perfectly fit with their ecological requirements as reported in Komárek and Anagnostidis (2005) and Komárek (2013). Some of them are Calothrix elenkinii, Geitlerinema jasorvense, G. splendidum, Jaaginema subtilissimum, Limnothrix redekei, Oscillatoria acuminata, Phormidium acuminatum, $P$. carboniciphilum, P. okenii, P. terebriforme, Symploca dubia and Westiellopsis prolifica. Broadly, this comprehensive new study updated and enriched the freshwater Egyptian algal flora by adding 6 new algal taxa for the first time: Symploca dubia, Westiellopsis prolifica, Euastrum insulare, Penium margaritaceum and Zygnemopsis sp. and Nitellopsis obtusa (based on the surveyed reference literature of Shaaban, 1994; Hamed, 2005, 2008 on Egyptian freshwater algal flora). It is also evident from the present pattern of species composition that some taxa are considered excellent bio-indicators of water pollution and eutrophication. Among them, non-heterocytous cyanoprokaryotes Oscillatoria limosa, $O$. princeps and $O$. tenuis mightily linked to relatively high accessibility of nitrates (Cantonati et al., 1996; Nowicka-Krawczyk and Żelazna-Wieczorek, 2013). Using of biota, particularly algal biocenosis, for monitoring springs and other identical environments, reflects the combined effects of the aquifer groundwater quality and anthropogenic impacts on these isolated systems (Williams 1991b). Macioszczyk and Dobrzyński (2002) and Cantonati et al. (2007a) discussed increased contents of minerals, cations and anions, specifically nitrate, nitrite and SRP, as remarkable indices of external contamination. According to the saprobien system of Kolkwitz and Marsson (1909) and its modifications (Zelinka and Marvan, 1961; Slàdeček, 1972; Villegas and de Giner, 1973; Makrushin, 1974), algal flora of El-Farafra Oasis was mainly composed of oligosaprobous forms (e.g. Vaucheria geminata and $V$. sessilis) with presence of some $\beta$-mesosaprobic tolerants (e.g. Oscillatoria limosa, O. princeps, Euglena acus and Coelastrum microporum), and a little contribution of $\alpha$-mesosaprobic biomonitors (e.g., Oscillatoria tenuis and Euglena oxyuris) with rarely polysaprobic feature of Anabaena constricta.

Some algal taxa recorded in this inventory are considered to be crenophilous (living mainly in springs), e.g. Geitlerinema jasorvense and Phormidium carboniciphilum (Komárek and Anagnostidis, 2005) to crenoxenic (living occasionally in springs), e.g. Jaaginema subtilissimum and Phormidium corium (Komárek and Anagnostidis, 2005), Westiellopsis prolifica (Anagnostidis and Komárek, 1990) and Zygnemopsis sp. (Randhawa, 1959; Aguilera et al., 2010). Concerning scarcity of algal diversity in tanks of the DWTPs, it might be devoted to light restriction (Neilson and Lewin, 1974).

Egyptian J. of Phycol. Vol. 16, $2015 \quad$ - 66 - 
Regarding to relationships between the environmental variables and algal communities, there is a positive relationship between cyanoprokaryota (mostly representing springs and wells) and the water temperature. By referring to this observation, a similar picture has been drawn by Mohamed and Al-Shehri (2008), Cantonati et al. (2012b) and Ward et al. (2012). The negative correlation between this group and $\mathrm{NO}_{3}-\mathrm{N}, \mathrm{NO}_{2}-\mathrm{N}$ and SRP concentrations was also confirmed by Yusoff and McNabb (1997) and Mohamed and Al-Shehri (2008). The high concentrations of these nutrients, particularly nitrates and nitrites, make the environment less suitable for flourishing of cyanobacteria, especially heterocytous forms. Contrary, euglenoids prefer inorganic and organic polluted habitats rich in nitrates and nitrites as a kind of growth mechanism (Kim and Boo, 2001; Wolowski and Hindák, 2005). Thus, this group was only restricted to lakes and agricultural drainage in this study. Members of chlorophyta are also mainly present in the previously-mentioned two habitats, where they like alkaline $\mathrm{pH}$ nature and this is in agreement with the findings of van Vuuren and Pieterse (2005) and Mishra et al. (2009). The fact that charophyte Nitellopsis obtusa was recorded only in winter season (where water temperature $=20^{\circ} \mathrm{C} ; \mathrm{pH}$ $=8.63$ ) at the agricultural water-fed ditch in close to Ain El-Balad spring accords with the findings of Caisová and Gąbka (2009), charophytes usually prefers alkaline water type with a $\mathrm{pH}$ limit ranges from 5 to 9.5 .

Highly remarkable appearance of cyanoprokaryotes on using both Chu\#10 and modified BG-11 media confirmed their validity as good culturing media for this group (Andersen et al., 2005), with respect to the other two used culturing media (Kolkwitz's solution and BBM). Absence of special micronutrients in Kolkwitz's solution or difference in nutrients concentrations in Bold Basal medium (BBM) could be explained as the main drivers. Culturing techniques not only permitted to discover the cryptic algal taxa present in viable spores, but also allowed to follow and confirm the complete life cycle of the stigonematalean true T-branched cyanoprokaryote Westiellopsis prolifica. Members of stigonematalean cyanoprokaryotes are distributed all over the world, but are present highly in strictly special extreme biotopes e.g. springs (Anagnostidis and Komárek, 1990; Kaštovský and Johansen, 2008; Komárek, 2013; Nowicka-Krawczyk and Żelazna-Wieczorek, 2013).

Finally, springs and drilled wells of El-Farafra Oasis are not only considered to be strategic Egyptian freshwater resources having important urban and economical values, but also are locus classicus of new Egyptian freshwater algal taxa.

\section{Acknowledgements}

This work was partly funded in 2011 by a grant from the Egyptian National Commission for Education, Science and Culture (UNESCO-ALECSO-ISESCO). Authors are also deeply grateful to Mr. El-Sayed Atia Younis, General Manager 
of Agriculture Ministry in the New Valley governorate (Egypt) for his kind help during materials sampling.

\section{References}

Abd El-Ghani, M. M. and Fawzy, A. M. (2006). Plant Diversity around springs and wells in five oases of the Western Desert, Egypt. International Journal of Agriculture and Biology, 8(2): 249-255.

Aboal, M.; Puig, M. A. and Prefasi, M. (1998). Diatom assemblages in springs in Castellón province, Eastern Spain. Archiv für Hydrobiologie, Supplement Algological Studies, 90: 79-95.

Aguilera, Á.; Souza-Egipsy, V.; González-Toril, E.; Rendueles, O. and Amils, R. (2010). Eukaryotic microbial diversity of phototrophic microbial mats in two Icelandic geothermal hot springs. International Microbiology, 13(1):2132.

Ali, A. A. (2002). Hydrogeological studies on the Nubia Sandstone Aquifer in Bahariya and Farafra depressions, Western Desert, Egypt. Ph.D. Thesis, Fac. of Sci., Ain Shams Uni., 164 pp.

Amarouche-Yala, S.; Benouadah, A.; Bentabet, A. and López-García, P. (2014). Morphological and phylogenetic diversity of thermophilic cyanobacteria in Algerian hot springs. Extremophiles, 18(6): 1035-1047.

Anagnostidis, K. and Komárek, J. (1990). Modern approach to the classification system of Cyanophytes 5. Stigonematales. Algological Studies, 59: 1-73.

Andersen, R. A.; Berges, J. A.; Harrison, P. J. and Watanabe, M. M. (2005). Appendix A: Recipes for Freshwater and Seawater Media. In: Algal culturing techniques. Andersen, R. A. (ed.), Elsevier Academic Press, Inc, 429-538 pp.

APHA (2005). Standard Methods for the Examination of Water and Wastewater, $20^{\text {th }}$ edition - American Public Health Association, Washington, DC.

Ayyad, M. A. and Ghabbour, S. I. (1986). Hot deserts of Egypt and the Sudan. In: Ecosystems of the World 12B, Hot Deserts and Arid Shrublands. Evenari, M.; Noy-Meir, L. and Goodall, D. W. (eds.), Elsevier Pub., Amsterdam, 149202 pp.

Bedir, S. E. (2014). Detection of cyanobacterial harmful algae in drinking water plants. International Water Technology Journal, 4(2): 81-89.

Bellinger, E. G. and Sigee, D. C. (2010). Sampling, biomass estimation and counts of freshwater algae. In: Freshwater Algae: Identification and use as Bioindicators. John Wiley \& Sons, Ltd, UK, 41-97 pp.

Bornkamm, R. and Kehl, H. (1990). The plant communities of the Western Desert of Egypt. Phytocoenologia, 19: 149-231.

Caisová, L. and Gąbka, M. (2009). Charophytes (Characeae, Charophyta) in the Czech Republic: taxonomy, autecology and distribution. Fottea, 9(1): 1-43.

Cantonati, M.; Angeli, N.; Bertuzzi, E. and Spitale, D. (2012a). Diatoms in springs of the Alps: spring types, environmental determinants, and substratum. Freshwater Science, 31(2): 499-524. 
Cantonati, M.; Angeli, N.; Virtanen, L.; Wojtal, A. Z.; Gabrieli, J.; Falasco, E.; Lavoie, I.; Morin, S.; Marchetto, A.; Fortin, C.; Smirnova, S. (2014). Achnanthidium minutissimum (Bacillariophyta) valve deformities as indicators of metal enrichment in diverse widely-distributed freshwater habitats. Science of The Total Environment, 475: 201-215.

Cantonati, M.; Decet, F.; Corradini, F. and Bertuzzi, E. (2007a). The significance of chemical and physical factors influence the ecology of springs, and a case study in the south-eastern Alps (Dolomiti Bellunesi National Park). In: The spring habitat: biota and sampling methods. Cantonati, M.; Bertuzzi, E. and Spitale, D. (eds.), Museo Tridentino di Scienze Naturali, Trento. Monografie del Museo Tridentino di Scienze Naturali, 4: 45-76 pp.

Cantonati, M.; Füreder, L.; Gerecke, R.; Jüttner, I.; Cox, E. (2012c). Crenic habitats, hotspots for freshwater biodiversity conservation: toward an understanding of their ecology. Freshwater Science, 31: 463-480.

Cantonati, M.; Gerecke, R. and Bertuzzi, E. (2006). Springs of the Alpssensitive ecosystems to environmental change: From biodiversity assessments to long-term studies. In: Developments of Hydrobiology, Ecology of high altitude aquatic systems in the Alps. Lami, A. and Boggero, A. (eds.), Hydrobiologia, 562: 59-96.

Cantonati, M.; Komárek, J. and Zurita, G. A. M. (2015). Cyanobacteria in ambient springs. Biodiversity and Conservation, 24(4): 865-888.

Cantonati, M.; Rott, E. and Pipp, E. (1996). Ecology of cyanophythes in mountain springs of the River Sarca catchment (Adamello-Brenta Regional Park, Trentino, Northern Italy). Algological Studies, 83:145-162.

Cantonati, M.; Rott, E.; Pfister, P. and Bertuzzi, E. (2007b). Benthic algae in springs: Biodiversity and sampling methods. In: The spring habitat: biota and sampling methods. Cantonati, M.; Bertuzzi, E. and Spitale, D. (eds.), Museo Tridentino di Scienze Naturali, Trento. Monografie del Museo Tridentino di Scienze Naturali, 4: 77-112 pp.

Cantonati, M.; Rott, E.; Spitale, D.; Angeli, N. and Komárek, J. (2012b). Are benthic algae related to spring types?. Freshwater Science, 31(2): 481-498.

Carroll, D. (1962). Rainwater as chemical agent of geologic process: A review. U.S. geological survey water-supply paper, $1535-G, 18$ pp.

Chapman, H. D. and Pratt, P. F. (1978). Water analysis. In: Methods of analysis for soil, plants and water. Division of Agricultural Sciences, University of California, 60-65 pp.

De Desenko-Schegolova, N. T. and Gollerbach, M. M. (1962). Freshwater algae of USSR. Vol. 5. Xanthophyta. Pub. Acad." Sov. Nauke" USSR, Moscow, Leningrad. 272 pp.

De Desenko-Schegolova, N. T.; Matvianko, A. M. and Shkorbatov, D. A. 
(1959). Freshwater algae of USSR. Vol. 8. Chlorophyta: Volvocineae. Pub. Acad. "Sov. Nauke" USSR, Moscow, Leningrad, 230 pp.

Desikachary, T. V. (1959). Cyanophyta. Indian Council of Agricultural Research, New Delhi, 686 pp.

Egyptian Higher Committee for Water "EHCW". (2007). Egyptian standards for drinking and domestic uses. Health and Population Minister's decree No. (458), central department for environmental affairs, Ministry of Health and Population.

El Sabri, M. A. and El Sheikh, A. E. (2009). Groundwater sustainability of the Post Nubian Sandstone Aquifer in Farafra Oasis, Western Desert, Egypt. Assiut Univ. Journal of Geology, 8(2): 1-32.

El-Bastawesy, M. and Ali, R. R. (2011). The use of GIS and remote sensing for the assessment of waterlogging in the dryland irrigated catchments of Farafra Oasis in Egypt. Hydrology and Earth System Sciences Discussions (HESS), 8:10535-10563.

El-Sebaie, O. D.; Abd El-Kerim, G. E.; Ramadan, M. H.; Abd El-Atey, M. M. and Taha, S. A. (2002). Water treatment plants assessment at Talkha power plant. Journal of Egyptian Public Health Association, 77(3-4): 347-370.

Erman, N. A. (2002). Lessons from a long-term study of springs and spring invertebrates (Sierra Nevada, California, U.S.A.) and implications for conservation and management. In: Proceedings of the meeting on spring-fed wetlands : important scientific and cultural resources of the intermountain region. Sada, D. W. and Sharpe, S.e. (eds.), Las vegas, Nevada. Desert Research Institute, Reno, Nevada, 1-13 pp.

Fawzy, M. A.; Hifney, A. F.; Issa, A. A. and Ghareib, G. (2013). Phytochemical constituents and allelopathic effects of some medicinal plants extract on the soil algal diversity. Journal of Agricultural Science and Technology, A3:1000-1009.

Gharib, G. E. (2004). Ecological studies on algal communities in relation to environmental factors at El-Kharga oasis, New Valley, Egypt. M.Sc. Thesis. Fac. of Sci., El-Minia Uni., 115 pp.

Glazier, D. S. (2009). Springs. In: Encyclopedia of Inland Waters. Vol. 1. Likens, G. E. (ed.), Academic Press Elsevier, 734-75.

Gollerbach, M. M.; Kosinckaja, E. K. and Polanskii, V. I. (1953). Freshwater algae of USSR, Vol. 2. Cyanophyta. Pub. "Sov. Nauke" Moscow, 652 pp.

Guillard, R. R. L. (2005). Purification Methods for Microalgae. In: Algal Culturing Techniques. Andersen, R. A. (ed.), Elsevier Academic Press, Inc, 117-132 pp.

Hamed, A. F . (2005). Survey of distribution and diversity of blue-green algae (Cyanobacteria) in Egypt. Acta Botanica Hungarica, 47: 117-136.

Hamed, A. F. (2008). Biodiversity and distribution of blue-green algae/cyanobacteria and diatoms in some of the Egyptian water habitats in

Egyptian J. of Phycol. Vol. 16, $2015 \quad$ - 70 - 
relation to conductivity. Australian Journal of Basic and Applied Sciences, 2(1): 1-21.

Hifney, A. F.; Adam, M. S.; Gharib, G.; Issa, A. A. (2013). Allelopathic effects of some weeds on rhizosphere algae at El-Kharga Oasis (New Valley), Egypt. Journal of Biology and Earth Sciences, 3(1): B42-B53.

http://en.wikipedia.org/wiki/Farafra,_Egypt; searched on $1^{\text {st }}$ February 2015.

Ibraheem, I. B. M. and Al-Sherif, E. A. (2009). Distribution of the flowering plants and cyanobacteria in relation to soil characters in Bahariya oasis, Egypt. International Journal of Botany, 5(1): 36-46.

Imam, A. I. (2007). Evaluation of some integrated cotton pest managements under El-Farafra Oasis conditions. Ph.D. Thesis. Fac. of Sci. Ain Shams Uni., 229 pp.

Kamal El-Din, M. M.; Wheeler, E. A. and Bartlett, J. A. (2006). Cretaceous woods from the Farafra Oasis, Egypt. International Association of Wood Anatomists (IAWA) Journal, 27 (2): 137-143.

Kaštovský, J. and Johansen, J. R. (2008). Mastigocladus laminosus (Stigonematales, Cyanobacteria): phylogenetic relationship of strains from thermal springs to soil-inhabiting genera of the order and taxonomic implications for the genus. Phycologia, 47: 307-320.

Kaštovský, J.; Gomez, E. B; Hladil, J. and Johansen, J. R. (2014). Cyanocohniella calida gen. et sp. nov. (Cyanobacteria: Aphanizomenonaceae) a new cyanobacterium from the thermal springs from Karlovy Vary, Czech Republic. Phytotaxa, 181(5): 279-292.

Keleher, M. J. And Rader, R. B. (2008). Dispersal imitations and history explain community composition of metaphyton in desert springs of the Bonneville Basin, Utah: A multiscale analysis. Limnology and Oceanography, 53: 1604-1613.

Khalil, M. A.; Salem, Z. E.; Gheda, S. F. and El-Sheikh, M. M. (2013). Quality assessment of drinking water in Tanta City, Egypt. Journal of Environmental Science and Engineering, B2: 257-275.

Kim, J. T. and Boo, S. M. (2001). The relationships of green euglenoids to environmental variables in Jeonjucheon, Korea. Korean Journal of Limnology, 34(2): 81-89.

Kociolek, J. P. and Stoermer, E. F. (2009). Oligotrophy: The forgotten end of an ecological spectrum. In: Proceedings of the $20^{\text {th }}$ International Diatom Symposium (Dubrovnik, Croatia). Acta Botanica Croatica, 68: 465-472.

Kolkwitz, R. (1922). Appendix III: Culture media for algae. In: The cultivation of algae. Venkataraman, G. S. (ed.), T. S. Pruthi Pub. (1969), Indian Council of Agricultural Research, New Delhi, 237-246 pp.

Kolkwitz, R. and Marsson, M. (1909). Ökologie der tierischen Saprobien. Beiträge zur Lehre von der biologischen Gewässerbeurteilung. Internationale Revue der gesamten Hydrobiologie und Hydrographie, 2(1-2): 126-152.

Egyptian J. of Phycol. Vol. 16, 2015 
Komárek, J. (2013). Cyanoprokaryota. 3. Teil/ $3^{\text {rd }}$ Part: Heterocytous genera. In: Süsswasserflora von Mitteleuropa. Büdel, B.; Gärtner, G.; Krienitz, L. and Schagerl, M. (eds.). Elsevier/Spektrum, Heidelberg, 1130 pp.

Komárek, J. and Anagnostidis, K. (2005). Cyanoprokaryota, $2^{\text {nd }}$ part: Oscillatoriales. In: Süßwasserflora von Mitteleuropa. Büdel, B.; Krienitz, L.; Gärtner, G. and Schagerl, M. (eds.), Band 19/2. Elsevier - Spektrum Akademisher Verlag, München, 759 pp.

Kovach, W. L. (2000). MVSP - A MultiVariate Statistical Package for Windows, ver. 3.12b. Kovach Computing Services, Pentraeth, Wales, U.K.

Lowe, W. H. and Likens. G. E. (2005). Moving headwater streams to the head of the class. Bioscience, 55:196-197.

Macioszczyk, A. and Dobrzyński, D. (2002). Hydrogeochemia, strefy aktywnej wymiany wód podziemnych. Wydawnictwo Naukowe PWN, Warszawa, 448 pp.

Makrushin, A. V. (1974). Biological analysis of water quality. Acad. Sci. USSR. Zoological Inst. Leningrad, 246 pp.

Mishra, R. K.; Shaw, B. P. and Sahu, B. K. (2009). Seasonal appearance of Chlorophyceae phytoplankton bloom by river discharge off Paradeep at Orissa coast in the Bay of Bengal. Environmental Monitoring and Assessment, 149(14): 261-273.

Mohamed, Z. A. and Al-Shehri, A. M. (2008). Cyanobacteria of surface and ground waters in Asir region with new records to Kingdom of Saudi Arabia. Journal of King Abdulaziz University, Science, 20(2): 113-129.

Neilson, A. H. and Lewin, R. A. (1974). The uptake and utilization of organic carbon by algae: an essay in comparative biochemistry. Phycologia, 13(3):227-264.

Nowicka-Krawczyk, P. B. and Żelazna-Wieczorek, J. (2013). Cyanobacteria microflora in a limestone spring (Troniny spring, Central Poland). Acta Societatis Botanicorum Poloniae, 8(3): 219-224.

Philipose, M. T. (1967). Chlorococcales. Indian Council of Agricultural Research, New Delhi, 363 pp.

Piatek, J.; Piatek, M.; Zeeb, B. and El Shahed, A. (2009). Chrysophyte stomatocysts in Africa: the first description of an assemblage in the recent sediments of a thermo-mineral spring in Egypt. Phycologia, 48(1): 13-23.

Poulíčková, A.; Bogdanová, K.; Hekera, P. and Hájková, P. (2003). Epiphytic diatoms of the spring fens in the flysh area of the Western Carpathians. Biologia, 58(4): 749-753.

Puchnarewicz, E. (2006). Development in the desert: A case study of Farafra Oasis (Egypt). Africana Bulletin, 54: 252-255.

Randhawa, M. S. (1959). Zygnemataceae. Indian Council of Agricultural Research Pub., New Delhi , India, 478 pp.

Rosati, M.; Cantonati, M.; Primicerio, R. and Rossetti, G. (2014). Biogeography and relevant ecological drivers in spring habitats: A review on 
ostracods of the Western Palearctic. International Review of Hydrobiology, 99: 409-424.

Saafan, T. A.; Moharram, S. H.; Gad, M. I. and KhalafAllah, S. (2011). A multi-objective optimization approach to groundwater management using genetic algorithm. International Journal of Water Resources and Environmental Engineering, 3(7): 139-149.

Saber, A. A. (2010). Algal biodiversity of Rosetta branch of River Nile. M.Sc. Thesis, Fac. of Sci., Ain Shams Uni., Cairo, Egypt, 295 pp.

Sawyer, C. N. and McCarty, P. L. (1967). Chemistry for sanitary engineers, $2^{\text {nd }}$ edition, McGraw-Hill, New York, 518 pp.

Scarsbrook, M. R.; Barquín, J. and Gray, D. P. (2007). New Zealand cold water springs and their biodiversity. Science for conservation 278. Science and Technical Publishing, Department of Conservation, Wellington, 72 pp.

Shaaban, A. S. (1985). The algal flora of Egyptian oases. II- On the algae of Siwa oasis. In: Proceedings of Egyptian Botanical Society, 4: 1-10.

Shaaban, A. S. (1994). Freshwater algae of Egypt. UN Environmental Program, National Biodiversity Unit. Biological Diversity of Egypt. GF/6105-92-022205, 150 pp.

Shaaban, A. S. and El Habibi, A. (1978). The algal flora of Egyptian oases. IThe algal flora of Kharga oasis. Bulletin of Desert Institute, 28(1): 227-232.

Shaaban, A. S. and Hamed, A. F. (1997). Freshwater algae of El-Arish Valley and its vicinity (North Sinai), Egypt. The Desert Inst. Bull., 47: 101-118.

Shaaban, A. S.; Hamed, A. F. and Fumanti, B. (1997). The algal flora of Egyptian oases. III- The algal flora of the thermal springs of Bahariya oasis. Egyptian Journal of Aquatic Biology and Fisheries, 1(1): 85-98.

Sherwood, A. R. and Sheath, R. G. (1999). Seasonality of epilithic diatoms and macroalgae from two spring-fed Texas springs. Hydrobiologia, 390:73-82.

Slàdeček, V. (1972). The vector of saprobity and the system of water quality. Polskie Archiwum Hydrobiologii, 19 (2); 211-213.

Soyer, J. (1970). Bionomie benthique du plateau continental de la cote catalane Francaise. III. Les peuplements de Copepodes harpacticoides (Crustacea). Vie et Milieu, 21: 337-511.

Spitale, D.; Leira, M.; Angeli, N. and Cantonati, M. (2012). Environmental classification of springs of the Italian Alps and its consistency across multiple taxonomic groups. Freshwater Science, 31(2): 563-574.

Uher, B.; Skácelová, O. and Kováčik, L. (2001). Sinice několika studánek v okolí Brna (Cyanobacteria of several wells in Brno surroundings). Czech Phycology, Olomouc, l: 21-30.

van der Kamp, G. (1995). The hydrogeology of springs in relation to the biodiversity of spring fauna: a review. Journal of the Kansas Entomological Society, 68 (2): 4-17.

van Vuuren, S. J. and Pieterse, A. J. H. (2005). The use of multivariate 
analysis as a tool to illustrate the influence of environmental variables on phytoplankton composition in the Vaal River, South Africa. African Journal of Aquatic Science, 30(1): 17-28.

Villegas, J. and de Giner, G. (1973). Phytoplankton as biological indicator of water quality. Water Research, 7 (3): 479-483.

Ward, D. M.; Castenholz, R. W. and Miller, S. R. (2012). Cyanobacteria in Geothermal Habitats. In: Ecology of Cyanobacteria II: Their Diversity in Space and Time. Whitton, B. A. (ed.), Springer Science+Business Media B.V., London, 39-63 pp.

West, W. and West, G. S. (1971a). A monograph of the British Desmidiaceae. Vol. I. London. First reprint by John Reprint Cooperation, USA, 224 pp.

West, W. and West, G. S. (1971b). A monograph of the British Desmidiaceae. Vol. II. London. First reprint by John Reprint Cooperation, USA, 204 pp.

West, W. and West, G. S. (1971c). A monograph of the British Desmidiaceae. Vol. III. London. First reprint by John Reprint Cooperation, USA, 274 pp.

Williams, D. D. (1991a). Life history traits of aquatic arthropods in springs. Memoirs of the Entomological Society of Canada, 155: 63-87.

Williams, D. D. (1991b). The spring as an interface between groundwater and lotic faunas, and as a tool in assessing groundwater quality. Verhandlungen der Internationalen Vereinigung für theoretische und angewandte Limnologie, 24: 1621-1624.

Wolowski, K. and Hindák, F. (2005). Atlas of Euglenophytes. VEDA, Hause of the Slovak Academy Pub., Bratislava, 136 pp.

World Health Organization "WHO". (2011). Acceptability aspects: taste, odour and appearance. In: guidelines for drinking water quality, $4^{\text {th }}$ edition. Geneva, Switzerland, 219-230 pp.

Yusoff, F. M. and McNabb, C. D. (1997). The effects of phosphorus and nitrogen on phytoplankton dominance in tropical fish ponds. Aquaculture Research, 28(8): 591-597.

Zabelina, M. M.; Kicelov, I. A.; Proschkina-Laverenko, A. I. and Scheshykova, V. S. (1951). The Freshwater Algae of USSR. Vol. 4. Bacillariophyta. Pub. "Sov. Nauke" Moscow, 619 pp.

Zelinka, M. and Marvan, P. (1961). Zur Präzisierung der biologischen Klassifikation der Reinheit fließender Gewässer. Archiv fur Hydrobiologie, 57: 389-407. 